\title{
Re-sleeping after reversal of remimazolam by flumazenil
}

\author{
Tomohiro Yamamoto $^{1}\left[\right.$ Miyuki Kurabe ${ }^{1} \cdot$ Yoshinori Kamiya $^{1}$ \\ Received: 17 February 2021 / Accepted: 1 March 2021 / Published online: 9 March 2021 \\ (c) Japanese Society of Anesthesiologists 2021
}

Keywords Re-sleeping $\cdot$ Remimazolam $\cdot$ Reversal $\cdot$ Flumazenil

\section{To the Editor:}

Remimazolam is reported to be a novel ultrashort-acting benzodiazepine [1]. It is highly advantageous as it induces faster and safer recovery than pre-existing intravenous and volatile anesthetics since its action can be completely antagonized by flumazenil [2-4]. According to the package insert, an initial dose of $12 \mathrm{mg} / \mathrm{kg} / \mathrm{h}$ should be given until the patient falls asleep, and general anesthesia is maintained by continuous administration at $1 \mathrm{mg} / \mathrm{kg} / \mathrm{h}$ [5]. This rate can be increased up to $2 \mathrm{mg} / \mathrm{kg} / \mathrm{h}$ and a maximum of $0.2 \mathrm{mg} /$ $\mathrm{kg}$ can be administered as a bolus, if necessary. However, a 62-year-old female patient $(152.4 \mathrm{~cm}, 57.9 \mathrm{~kg})$ became drowsy again in the ward $45 \mathrm{~min}$ after antagonizing remimazolam with flumazenil, despite following the administration method described in the instructions. Rocuronium $0.6 \mathrm{mg} /$ $\mathrm{kg}$ was additionally utilized for the endotracheal intubation. After the general anesthesia induction, remimazolam was administered during the surgery (laminoplasty for lumbar spinal stenosis) at $1 \mathrm{mg} / \mathrm{kg} / \mathrm{h}$ for $3.5 \mathrm{~h}$, and the intraoperative BIS value was maintained in the range of 40-60. Remimazolam was discontinued after postoperative X-ray, and $0.5 \mathrm{mg}$ flumazenil was administered. After confirming that the patient was well awake and could follow orders, she was sent to the ward. Once there, she became unconscious again. After the anesthesiologist examined the patient, a second dose of $0.5 \mathrm{mg}$ flumazenil was administered. The patient awakened instantly and was able to follow orders. After that, the patient did not fall into somnolence again.

The package insert of flumazenil states that its contextsensitive half-time is about $50 \mathrm{~min}$, and the package insert

\section{Tomohiro Yamamoto}

yamatomo@med.niigata-u.ac.jp;

yamatomo270@hotmail.com

1 Division of Anesthesiology, Niigata University Graduate School of Medical and Dental Sciences, 1-757, Asahimachi-dori, Chuo ward, Niigata 951-8510, Japan of flumazenil states that the active durations of flumazenil and remimazolam are comparable. Based on our experience, it is important to recognize the possibility of re-sleeping of patients after complete reversal of remimazolam by flumazenil, even when all recommended dosages are used.

Author contributions TY: wrote the first draft of the manuscript. MK: wrote the first draft of the manuscript. YK: wrote the first draft of the manuscript.

Funding None.

\section{Compliance with ethical standards}

Conflict of interest The authors declare that they have no conflict of interest statement.

\section{References}

1. Wesolowski AM, Zaccagnino MP, Malapero RJ, Kaye AD, Urman RD. Remimazolam: pharmacologic considerations and clinical role in anesthesiology. Pharmacotherapy. 2016;36:1021-7.

2. Doi M, Morita K, Takeda J, Sakamoto A, Yamakage M, Suzuki T. Efficacy and safety of remimazolam versus propofol for general anesthesia: a multicenter, single-blind, randomized, parallelgroup, phase IIb/III trial. J Anesth. 2020;34:543-53.

3. Doi M, Hirata N, Suzuki T, Morisaki H, Morimatsu H, Sakamoto A. Safety and efficacy of remimazolam in induction and maintenance of general anesthesia in high-risk surgical patients (ASA Class III): results of a multicenter, randomized, double-blind, parallel-group comparative trial. J Anesth. 2020;34:491-501.

4. Masui K. Remimazolam besilate, a benzodiazepine, has been approved for general anesthesia!! J Anesth. 2020;34:479-82.

5. Hirata N, Hayamizu K, Yamakage M. How to administer remimazolam for anesthesia induction. J Anesth. 2020;34:962.

Publisher's Note Springer Nature remains neutral with regard to jurisdictional claims in published maps and institutional affiliations. 